\title{
Screen-printed Electrochemical Sensor Based on Reduced Graphene doped Poly (L-dopa)/PEDOT:PSS Composites for Epinephrine Detection
}

\author{
Yile Hu, Hui Yang ${ }^{*}$, Wei Li, Keke Du, Simin Song, Wenhui Zhang, Zhiqiang Wei, Sanqiang Li* \\ College of Basic Medical, Henan University of Science and Technology, Luoyang, 471023, Henan, \\ China. \\ *E-mail: yanghui7761@163.com, sanqiangli2001@163.com
}

Received: 3 August 2021 / Accepted: 23 September 2021 / Published: 10 November 2021

\begin{abstract}
In current work, the highly sensitive screen-printed electrochemical sensor for epinephrine (EP) detection was constructed based on reduced graphene doped poly (L-dopa)/PEDOT:PSS composites by the simple solution coating method and electropolymerization. The constructed sensor exhibited excellent sensor performances and recorded the linear response of EP in the concentration range of 0.10$16.50 \mu \mathrm{M}$ with detection limit of $0.02 \mu \mathrm{M}$. The practical analytical utility provides great promise for sensitive measurements of EP in human serum, and has broad prospects in clinical and pharmaceutical applications.
\end{abstract}

Keywords: Reduced Graphene, Screen-printed Electrochemical Sensor, Poly (L-dopa), PEDOT:PSS, Epinephrine

\section{$\underline{\text { FULL TEXT }}$}

(C) 2021 The Authors. Published by ESG (www.electrochemsci.org). This article is an open access article distributed under the terms and conditions of the Creative Commons Attribution license (http://creativecommons.org/licenses/by/4.0/). 